\title{
Numerical resolution of a reinforced random walk model arising in haptotaxis
}

\author{
Ana I. Muñoz , J. Ignacio Tello
}

\section{Keywords:}

Haptotaxis

Parabolic PDE

ODE

Characteristics method

Finite element method

Stabilization of solutions

\begin{abstract}
A B S T R A C T
In this paper we study the numerical resolution of a reinforced random walk model arising in haptotaxis and the stabilization of solutions. The model consists of a system of two differential equations, one parabolic equation with a second order non-linear term (haptotaxis term) coupled to an ODE in a bounded two dimensional domain. We assume radial symmetry of the solutions. The scheme of resolution is based on the application of the characteristics method together with a finite element one. We present some numerical simulations which illustrate some features of the numerical stabilization of solutions.
\end{abstract}

\section{Introduction}

A characteristic feature of living organisms is that they respond to the environment in search of food and a reproductive mate, which is called taxis. Corresponding to the type of the external stimulus, various types of taxis are defined, such as haptotaxis, chemotaxis and others.

Chemotaxis is a process whereby living organisms respond to chemical substance by moving toward higher or lower concentrations of the chemical substance, or by aggregating or dispersing. Haptotaxis is closely related to chemotaxis, as it is the directional motility or growth of cells following gradient of cellular adhesion sites or substrate-bound chemoattractants. The gradient of the chemical signal in this case is expressed or bound on a surface, in contrast to the classical model of chemotaxis, in which gradient develops in a soluble fluid. These gradients are naturally present in the extracellular matrix of the body during process such as angiogenesis.

In the majority of the theoretical analysis the signal is transported by diffusion, convection or by some other means. The classical chemotaxis equation was introduced by [11], after [15], as the first model to describe the aggregation of slime mold amoebae due to an attractive chemical substance. The model involves the density distribution of the bacteria $u$ and the chemical concentration $v$ in a coupled system of partial differential equations

$$
\begin{aligned}
& u_{t}=\Delta u-\operatorname{div}(u \chi(v) \nabla v) \\
& v_{t}-\Delta v=g(u, v)
\end{aligned}
$$

where $u_{t}=\frac{\partial u}{\partial t}$ and $v_{t}=\frac{\partial v}{\partial t}$. 
However, in some chemotaxis phenomena, the diffusion of the chemical attractant is ignorable, the walker seems to modify the environment in a strictly local manner and there is little or no transport of the chemical substance.

In an attempt to gain understanding of the mechanisms that causes the aggregation of myxobacteria, which slide over slime trails thereby reinforcing the trails, [14] proposed a model based on reinforced random walks. The system of equations derived by Othmer and Stevens is the following:

$$
\begin{aligned}
& u_{t}=\operatorname{div}(D \nabla u-u \chi(v) \nabla v), \\
& v_{t}=g(u, v),
\end{aligned}
$$

where $D$ is the diffusion constant and $\chi(v)$ is the chemotactic sensitivity of the bacteria. Both, $\chi(v)$ and $g(u, v)$ depend on the nature of the interaction between the bacteria and the chemical stimulus.

[9] studied a reinforced random walk model in haptotaxis. They considered system 1,2 in a bounded domain $\Omega \subset R^{n}$ with boundary condition:

$$
\left(D \frac{\partial u}{\partial n}-u \chi(v) \frac{\partial v}{\partial n}\right)=0, \quad x \in \partial \Omega, \quad t>0
$$

where $\frac{\partial u}{\partial n}$ and $\frac{\partial v}{\partial n}$ are outward normal derivatives, the random motility $D$ is assumed to be constant and $\chi$ measures the haptotactic sensitivity. The function $g(u, v)$ is assumed to be of the form

$$
g(u, v)=\widetilde{h}(u, v) \phi(u, v)
$$

where, for some constants $0 \leqslant u_{1} \leqslant u_{2}, v_{1}<v_{2}$, it is satisfied

$$
\begin{aligned}
& \phi(u, v)>0, \quad \text { if } u_{1} \leqslant u \leqslant u_{2}, \quad v_{1} \leqslant v \leqslant v_{2}, \\
& \text { and } \widetilde{h}\left(u_{1}, v_{1}\right)=\widetilde{h}\left(u_{2}, v_{2}\right) .
\end{aligned}
$$

This problem describes the evolution of a biological species moving along a gradient of the concentration of a second species. Notice that in the case of chemotaxis systems, the second species diffuses in a higher or lower velocity, depending on the process, and it is modelized by a parabolic or elliptic equation.

This kind of equations, containing haptotaxis terms, arise for example in modeling cancer process, as angiogenesis, see for instance $[1,12]$. These problems also present mathematical challenges whereby several authors have been interested, as the literature shows, see for example $[9,13,16]$, and references therein.

From the mathematical point of view, in [9], it is proved that any stationary state $\left(u^{*}, v^{*}\right)$ of 1,2 is asymptotically stable provided:

$$
g=\widetilde{h} \phi, \quad \phi>0, \quad \widetilde{h}_{p}>0, \quad p \chi \widetilde{h}_{p}+\widetilde{h}_{w}<0 \quad \text { at } \quad\left(u^{*}, v^{*}\right) .
$$

If $(4)$ is satisfied, then any solution of 1,2 , in a bounded domain with boundary condition ( 3 ), and initial values near ( $u^{*}, v^{*}$ ), exists for all $t>0$ and converges as $t \rightarrow \infty$ to a nearby stationary solution $(\bar{u}, \bar{v})$. This assertion means that under assumption (4), solutions tend to an uniform distribution, provided the initial distribution is nearly uniform.

The question about what the behavior of solutions could be when condition (4) is not satisfied was the motivation for the study presented in this paper.

This paper is involved with the numerical resolution of a particular case of system 1,2 , considered in [9] as an example. To precise, we shall consider the following parabolic-ODE system posed in a bounded domain $\Omega \subset R^{2}$,

$$
\begin{aligned}
& u_{t}=\operatorname{div}\left(\nabla u-\left(u \frac{\beta}{\alpha+\beta v} \nabla v\right)\right), \quad x \in \Omega, \quad t>0, \\
& v_{t}=u-\mu h(v), \quad x \in \Omega, \quad t>0,
\end{aligned}
$$

that is, $\chi(\nu)=\frac{\beta}{\alpha+\beta \nu}$, with $\mu>0, \alpha>0$ and $\beta>0$, complemented with the boundary condition

$$
\left(\frac{\partial u}{\partial n}-u \frac{\beta}{\alpha+\beta v} \frac{\partial v}{\partial n}\right)=0, \quad x \in \partial \Omega, \quad t>0,
$$

and initial data

$$
u(x, 0)=u_{0}(x), \quad v(x, 0)=v_{0}(x), \quad x \in \Omega .
$$

For this particular case, if assumption

$$
\chi(v) h(v)<h^{\prime}(v) \text { for } u_{1} \leqslant u \leqslant u_{2}, v_{1} \leqslant v \leqslant v_{2}
$$

with $v_{2}-v_{1}$ small enough, is verified, then assumption (4) is also satisfied (see [9]). 
It is the purpose of this paper to solve numerically the system (5)-(8) in a bounded two dimensional domain when radial symmetry of solutions is assumed in order to answer in a way to the question about what could be the behavior of solution when (4) is not satisfied.

Regarding the numerical resolution of chemotaxis or haptotaxis models, it could be said that the finite-volume and finitedifference methods, or a combination of the two have been widely employed. These methods usually used a second order upwind scheme which is positivity preserving. These kind of methods are developed and used for example in [7]. In [10], it is used the method of lines to deal with taxis-diffusion-reaction models.

In Section 2, we describe the scheme of resolution, which is based on a combination of the characteristics method (as an alternative to the upwind technique) and the finite element method. The method here employed follows the ideas of [4]. In Section 3, we present some numerical results which illustrate the stabilization of solutions when (9), and therefore (4), is satisfied. We also show the results concerning two cases for which (9) is not satisfied (neither (4)). We shall see one simulation where the stabilization of solutions takes place and other where this does not occur. Finally, in Section 4 we comment some conclusions.

\section{Numerical analysis of the radially symmetrical case in $2 \mathrm{D}$}

This section deals with the numerical scheme we have employed to solve numerically the system (5)-(8) when it is assumed that solutions are radially symmetric. In this case, the problem reduces to one of only one spatial variable, which is the radius $r$. Without losing generality, we shall assume that $r \in[0,1]$.

The numerical resolution of the model and subsequence numerical simulations would allow us to study the stabilization of solutions.

To precise, the problem we intent to solve numerically in this section is the following:

$$
\begin{aligned}
& u_{t}=\frac{1}{r} \frac{\partial}{\partial r}\left(r\left(u_{r}-u \frac{\beta}{\alpha+\beta v} v_{r}\right)\right), \\
& v_{t}=u-\mu h(v),
\end{aligned}
$$

where $u_{r}=\frac{\partial u}{\partial r}$ and the same for $v$, complemented with appropriated initial and boundary conditions which will be mentioned later.

Due to the hyperbolic feature of Eq. (10), we opt for using a scheme which combines the method of characteristics and the reformulation of the convection term in (10) in terms of the total derivative with the finite element one (see [4,8] and references therein). We shall assume that the variables satisfy some regularity requirements that allow us to develop the numerical scheme. This technique has been successfully used in other fields (see for example $[2,5,6]$ ).

Next, we describe the numerical scheme. The equation for $u$, which in an expanded form is

$$
u_{t}=u_{r r}+\left(\frac{1}{r}-\frac{\beta v_{r}}{\alpha+\beta v}\right) u_{r}+\frac{\beta^{2} u}{(\alpha+\beta v)^{2}} v_{r}^{2}-\frac{\beta u}{r(\alpha+\beta v)} v_{r}-\frac{\beta u}{\alpha+\beta v} v_{r r}
$$

can be written in the following form,

$$
u_{t}+A u_{r}=u_{r r}+B
$$

where

$$
A=-\frac{1}{r}+\frac{\beta v_{r}}{\alpha+\beta v} \quad \text { and } \quad B=\frac{\beta^{2} u}{(\alpha+\beta v)^{2}} v_{r}^{2}-\frac{\beta u}{r(\alpha+\beta v)} v_{r}-\frac{\beta u}{\alpha+\beta v} v_{r r}
$$

Consider the total derivative $\frac{D u}{D t}$ defined as follows:

$$
\frac{D u}{D t}=u_{t}+A u_{r}+u \nabla \cdot A
$$

where $A$, which has been defined in (14), would be an artificial velocity field. Notice that

$$
\nabla \cdot A=\frac{1}{r} \frac{\partial}{\partial r}\left(r\left(\frac{\beta v_{r}}{\alpha+\beta v}-\frac{1}{r}\right)\right)=\frac{\beta v_{r}}{r(\alpha+\beta v)}+\frac{\beta v_{r r}}{\alpha+\beta v}-\frac{\beta^{2} v_{r}}{(\alpha+\beta v)^{2}}
$$

hence,

$$
\frac{D u}{D t}=u_{r r}+B+u \nabla \cdot A=u_{r r}
$$

Note that when $A$ is actually the velocity of an incompressible fluid, then $\nabla \cdot A$ is null. In this problem, $A$ is an artificial velocity and $\nabla \cdot A$ is not necessary null so we will have to take it into consideration and compute it. Next, we shall follow the notation and ideas presented in [4].

In our particular problem we shall consider the total derivative of $J u$, given by 


$$
\left.\frac{D J u}{D t}(\boldsymbol{r}, t)=\frac{\partial}{\partial \tau} J(r, t ; \tau) \boldsymbol{u}(X(r, t ; \tau), \tau)\right]_{\tau=t},
$$

where $J$ is the Jacobian associated with the change of coordinates from Eulerian to Lagrangian coordinates. The Jacobian $J(r, t ; \tau)$ of the change of coordinates is defined as follows

$$
J(r, t ; \tau):=1-\int_{\tau}^{t} \nabla \cdot A(X(r, t ; \tau), \tau) d \tau .
$$

Note that the presence of $J$ arises from the application of the characteristics method when one considers the conservative form for the convection term. We shall denote the characteristics $X$ by $X(r, t ; \tau)$, as $r$ and $t$ are parameters for the solution $X$ of

$$
\left\{\begin{array}{l}
\frac{d X(r, t ; \tau)}{d \tau}=A(X(r, t ; \tau), \tau) \\
X(r, t ; t)=r
\end{array}\right.
$$

which means that $X(r, t ; \cdot)$ should be the particle path that passes at $r$ at time $t$. The system (18) is backward in time but when $A$ is regular enough, say Lipschitz continuous, it has a unique solution.

Then, we obtain that

$$
\left.\frac{D J u}{D t}=J_{\tau} u+J\left(u_{r} X_{\tau}+u_{\tau}\right)\right]_{\tau=t}=u_{t}+\nabla(u A)
$$

where $J_{\tau}=\frac{\partial J}{\partial \tau}$ and the same for $X$ and $u$. Therefore, the problem we have to solve numerically for the variable $u$ turns out to be:

$$
\begin{aligned}
& \frac{D J u}{D t}=u_{r r}, \quad 0<t \leqslant T, \quad r \in(0,1), \\
& u(r, 0)=u_{0}(r), \quad r \in[0,1]
\end{aligned}
$$

where the initial condition $u_{0}(r)$ is assumed to be a regular function. Due to the diffusion term in (19) we need to prescribe boundary conditions. In this case, we consider the following boundary condition $u_{r}(0, t)=u_{r}(1, t)=0,0<t \leqslant T$. The problem for the variable $v$ is given by (11) complemented with the initial condition

$$
v(r, 0)=v_{0}(r), \quad r \in[0,1]
$$

with $v_{0}(r)$ also a regular function. In addition we shall assume that $v_{r}(0, t)=v_{r}(1, t)=0,0<t \leqslant T$, in the computation of the characteristics and $J$, which is in accordance with Eq. (11) and the boundary condition (7) of the original problem.

In order to solve (19) complemented with the corresponding initial data and boundary conditions, we shall use a time marching scheme. Hence, let $P>1$, be a natural number and let $\Delta t=T / P$ be the fixed time step of discretization. To discretize in time, we used the following formula for each $t^{n+1}=(n+1) \Delta t$, with $n=0, \ldots, P-1$ :

$$
\begin{aligned}
& \frac{D J u}{D t}\left(r, t^{n+1}\right) \simeq, \\
& \left.J^{n+1}(r) u\left(X\left(r, t^{n+1} ; t^{n+1}\right), t^{n+1}\right)-J^{n}(r) u\left(X\left(r, t^{n+1} ; t^{n}\right), t^{n}\right)\right] / \Delta t,
\end{aligned}
$$

where $J^{n}(r)=J\left(r, t^{n+1} ; t^{n}\right)$ and $J^{n+1}(r)=J\left(r, t^{n+1} ; t^{n+1}\right)$. Note that if we consider $t=t^{n+1}$ in (17), (18), we get that

$$
J^{n+1}=J\left(r, t^{n+1} ; t^{n+1}\right)=1, \quad X\left(r, t^{n+1} ; t^{n+1}\right)=r
$$

therefore (21) becomes

$$
\frac{D J u}{D t}\left(r, t^{n+1}\right) \simeq\left[u^{n+1}-J^{n}(r) u^{n}\left(X^{n}(r)\right)\right] / \Delta t,
$$

where $u^{n}(r)=u\left(r, t^{n}\right)$ and $X^{n}(r)=X\left(r, t^{n+1} ; t^{n}\right)$ is the solution of (18) that will be in $r$ at time $t^{n+1}$.

As a result of the discretization with respect to time, we have that for each step in time $t^{n+1}$ we have to solve a stationary problem. In particular we shall consider the following problem for the unknown $u^{n+1}(r)$ :

$$
\begin{aligned}
& u^{n+1}-\Delta t u_{r r}^{n+1}-J^{n}(r) u^{n}\left(X^{n}(r)\right)=0, \quad r \in(0,1), \\
& u_{r}^{n+1}(0)=u_{r}^{n+1}(1)=0,
\end{aligned}
$$

where $J^{n}(r)$ and $u^{n}\left(X^{n}(r)\right)$ are known as they have been computed in the previous step in time. The variational formulation corresponding to the problem (24), (25) is the following

$$
\int_{0}^{1}\left(u^{n+1} w-\Delta t u_{r}^{n+1} w_{r}-J^{n}(r) u^{n}\left(X^{n}(r)\right) w\right) d r=0, \quad \forall w \in H^{1}(0,1) .
$$


In order to discretize the problem (26) with respect to the spatial coordinate $r$, we use Lagrange linear finite elements. After applying some quadrature formula for integration and some algebraic operations, we obtain a linear system of equations for the unknowns $u_{i}^{n+1}=u^{n+1}\left(r_{i}\right)$, and $i=0,1,2, . . N$, being $\left\{r_{i}\right\}$ the partition of the domain $[0,1]$ considering $\Delta r$ as the step for the space discretization. The matrix of coefficients associated to the system is a band tri-diagonal one. The system is solved by using the Thomas algorithm.

Finally, once that we have updated the values for $u$, we pass to update the ones for $v$. In this case, we consider the following finite difference scheme,

$$
\frac{v_{i}^{n+1}-v_{i}^{n}}{\Delta t}=u_{i}^{n+1}-\mu h\left(v_{i}^{n}\right)
$$

therefore,

$$
v_{i}^{n+1}=v_{i}^{n}+\Delta t\left(u_{i}^{n+1}-\mu h\left(v_{i}^{n}\right)\right), \quad \text { for } \quad i=0,1,2, . . N .
$$
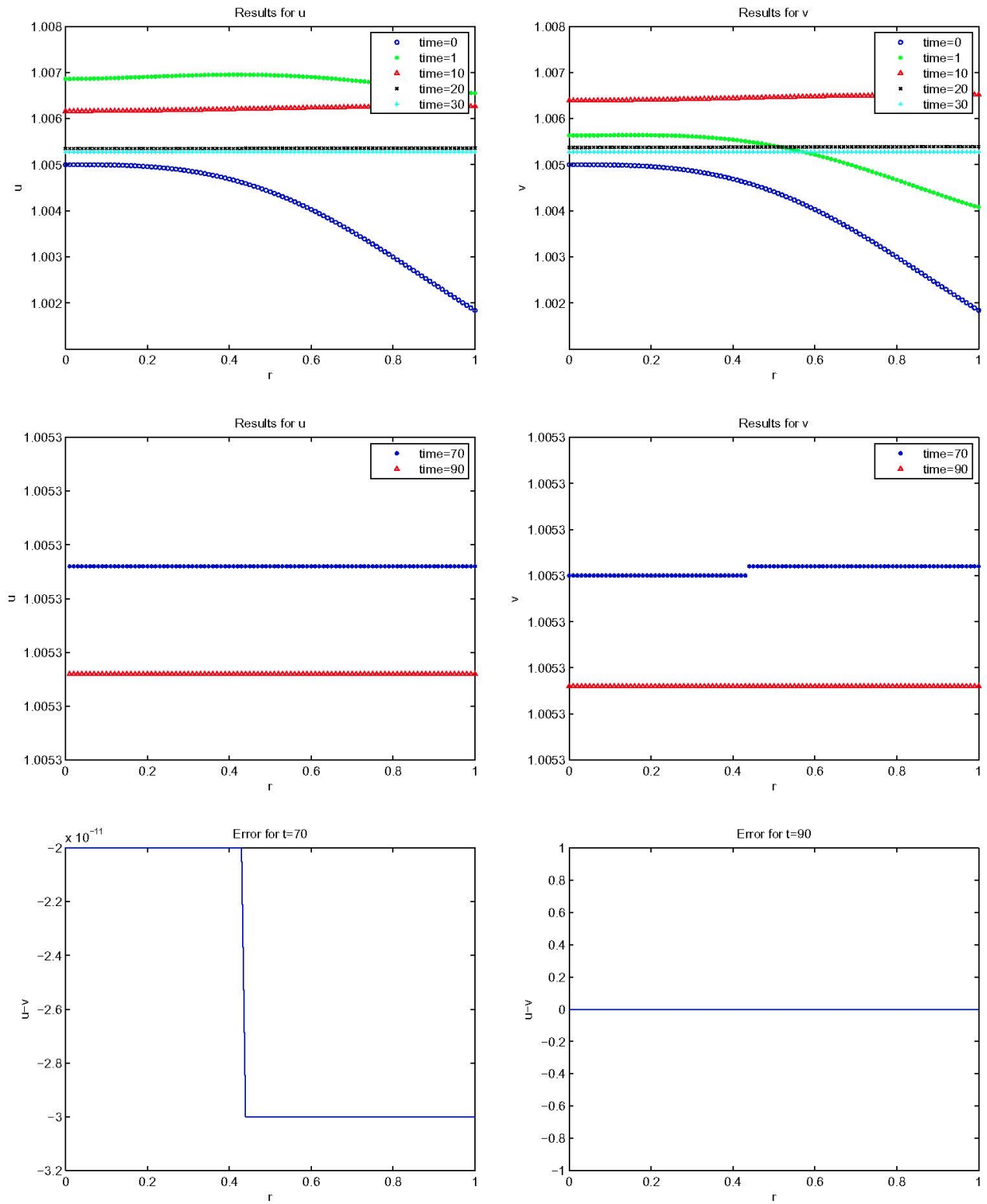

Fig. 1. Results obtained with $u_{0}(r)=v_{0}(r)=1+0.005 e^{-r^{3}}, \chi(v)=\frac{1}{1+v^{\prime}}, h(v)=v$ and $\mu=1$, that is $\nu_{t}=u-v$. In this case assumption (9) is satisfied. 


\section{Numerical simulations in the radially symmetric case}

In this section we shall present some numerical simulations obtained with the scheme of resolution described in the previous section in order to illustrate the numerical stabilization of solutions for different $h(v)$. It is the purpose of these simulations to show the well performance of the method as we are able to reproduce the expected behavior of solutions to the problem considered in [9], Example 1, when assumption (9) is satisfied, and on the other side to bring some insight about possible behaviors of solutions when it is not. In this occasion we are not mainly concerned with the obtention of biologically meaningful solutions not with modeling considerations. However, we want to highlight that the algorithm can be used in more general scenarios regarding the choices for the expressions of $\chi$ and $g(u, v)$ and therefore, it could provide an effective way of selecting expressions leading to desirable profiles for the solutions from the biomedical point of view.

The problem has been solved in the interval $r \in[0,1]$. The steps in time and space used in the simulations presented in Figs. 1, 3 and 4 are $\Delta t=10^{-4}$ and $\Delta r=10^{-2}$. It has been performed a great number of numerical simulations, regarding initial data and expressions for $\chi$ and $g(u, v)$, but for the sake of brevity we only present some of them concerned with the stabilization problem considered in this paper. We have chosen three cases based on the choice of $h(v)$. One case, presented in Fig. 1, illustrates how the stabilization of the solution takes place as expected when assumption (9) is satisfied. A second case, presented in Fig. 3, shows how stabilization of solutions is possible despite of the fact that assumption (9) is not satisfied. And finally, a third case, presented in Fig. 4, in which assumption (9) is not satisfied and the stabilization of the solution does not take place.

Regarding the initial data, we consider in all the simulations shown here the same $u_{0}$ and $v_{0}$, to precise

$$
u_{0}(r)=v_{0}(r)=1+0.005 e^{-r^{3}}
$$

but a number of simulations with different profiles for the initial data $u_{0}$ and $v_{0}$ have been performed. According to our numerical results we could conjecture that it is the expression of $h(v)$ the one which plays a determinant role in order to obtain the stabilization of solutions or not.

In Fig. 1, we present the numerical results obtained with $\chi(v)=\frac{1}{1+v}, \mu=1$ and $h(v)=v$. With this choice, assumption (9) is satisfied. One can observe that the stabilization of solution takes place, and therefore we obtain that after a certain time $T$ that $u=v=C(=1.005270584662)$ for $t \geqslant T$. We associate to each time $t$ an error defined in this case by $e(t, r)=u(t, r)-v(t, r)$ in order to know how far we are from the constant stationary values.

In order to illustrate the well performance of the numerical scheme we present the results obtained for $u$ and $v$ in the case considered in Fig. 1 for different steps in time and space for $t=0.1$ at $r=0$ and $r=1$ in Table 1 and 2, respectively. Notice that the solution is decreasing so the values are between the ones in $r=0$ and $r=1$.

Note also that the stability of the numerical scheme depends on $A$ (related to the convection term) whose values depend at the same time on the step of the discretization in space. The values of $A(t, r)$ do not change significantly with respect to time for fixed steps in time and space as it can be observed in Fig. 2 (left).

In Fig. 3, we present the numerical results obtained with the same initial data considered in Fig. 1, the same expression for $\chi, \mu=1$ and $h(v)=v+1$. In this case, assumption (9) is not satisfied, however the solution stabilizes to constant values which satisfy $u=v+1$, in particular these values are $u=0$ and $v=-1$. The variable error mentioned in the graphics is defined by $e(t, r)=u(t, r)-(v(t, r)+1)$.

In Fig. 4, we present the numerical results obtained with the same initial data and $\chi$ considered in Figs. 1 and 3 , just to show that the stabilization phenomenon mainly depends on the expression of $h(v)$. In this third case, $h(v)=e^{\frac{1}{v+1}}$ so assumption (9) is not satisfied, and we obtain the solution does not stabilize to constant values and even there is a time $t_{*}$, such that the numerical solution seems to present a kind of numerical blow up.
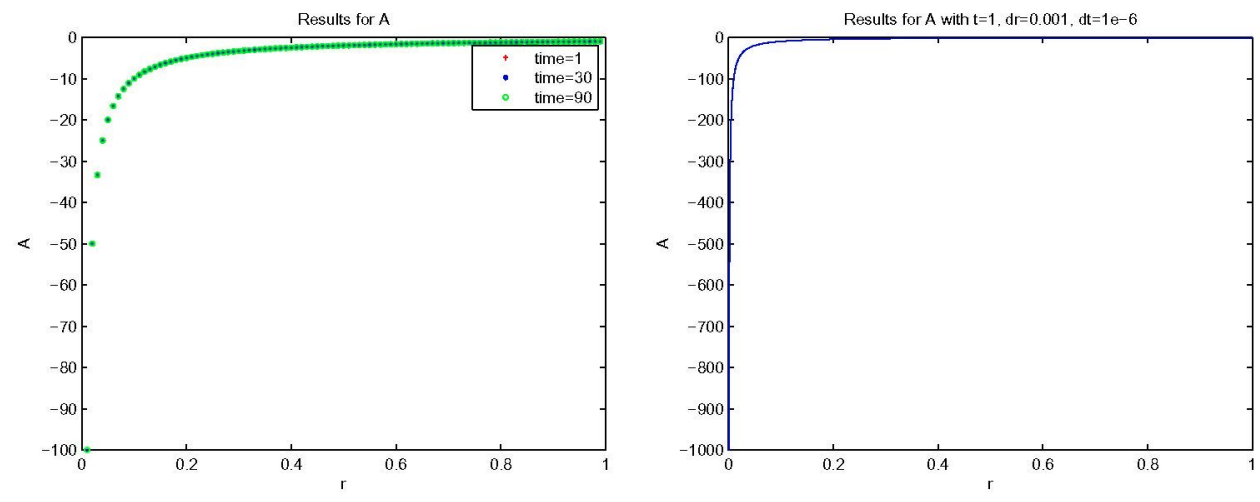

Fig. 2. Results obtained for $A=-\frac{1}{r}+\frac{v_{r}}{1+\nu^{*}}$. On the left we present the results obtained with $\Delta t=10^{-4}$ and $\Delta r=10^{-2}$ for $t=1, t=30$ and $t=90$. On the right we present the result obtained for $A$ with $\Delta t=10^{-6}$ and $\Delta r=10^{-3}$ for $t=1$. 

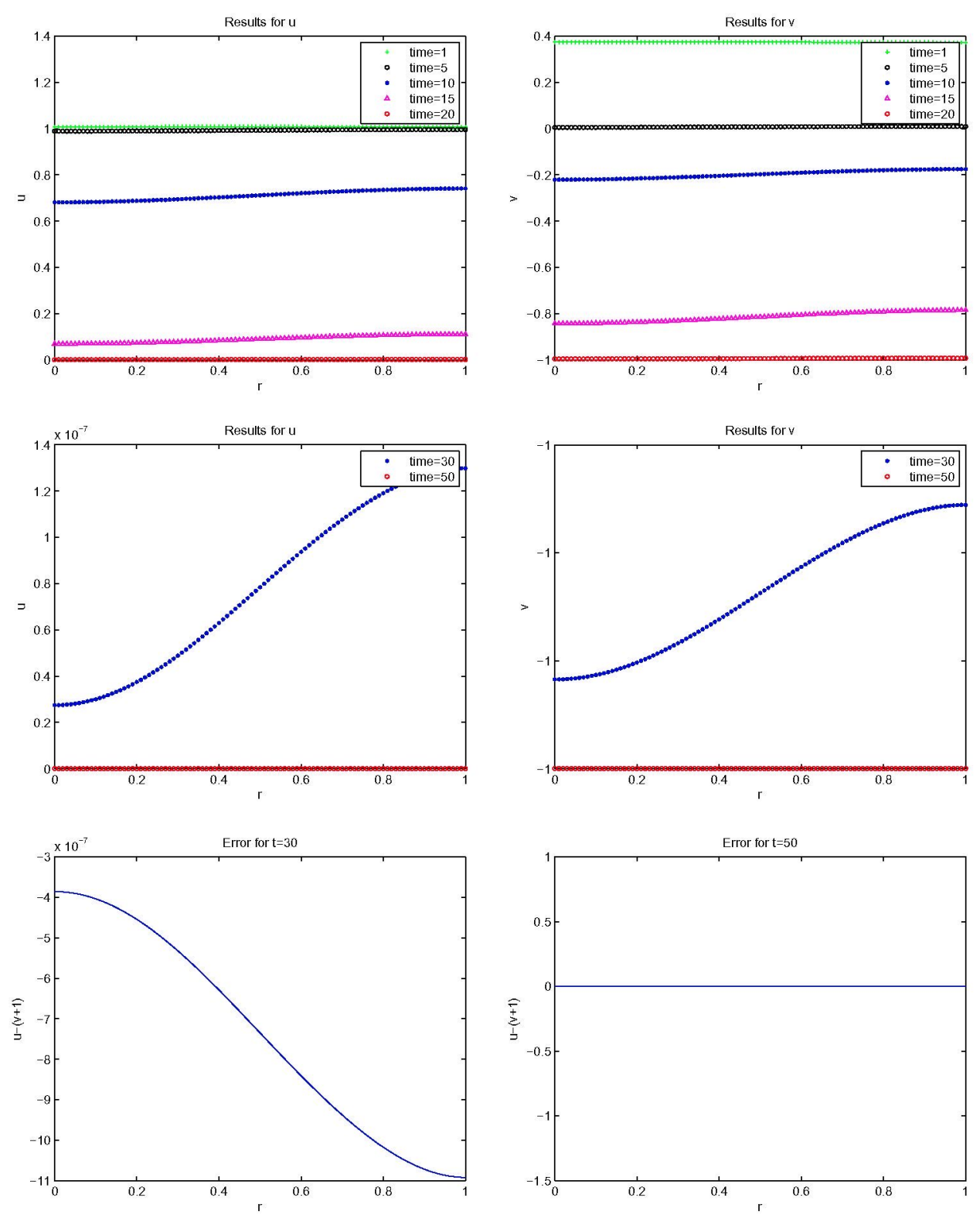

Fig. 3. Results obtained with $u_{0}(r)=v_{0}(r)=1+0.005 e^{-r^{3}}, \chi(v)=\frac{1}{1+\nu}, h(v)=v+\mathbf{1}$ and $\mu=\mathbf{1}$, that is $v_{t}=\boldsymbol{u}-(\nu+\mathbf{1})$. In this case assumption (9) is not satisfied.

\section{Discussion and conclusions}

In this paper we study the numerical resolution of a system of two differential equations, one parabolic equation with second order non-linear terms (haptotaxis) and an ODE. The problem describes the evolution of a biological species moving along a gradient of the concentration of a second species. The system is closely related with chemotaxis systems, where the second species diffuses in a higher or lower velocity depending on the process and it is modelized by parabolic or elliptic equations.

Similar systems, containing haptotaxis terms, are used to describe cancer processes, as angiogenesis (see for example $[1,12]$ ). The problem also presents mathematical challenges whereby several authors have been interested, as the literature shows, see for instance $[9,13,16]$, and references therein.

In [9], the system (1)-(3) is studied under assumption (4), and it is established that any stationary state $\left(\boldsymbol{u}^{*}, v^{*}\right)$ is asymptotically stable. If (4) is satisfied, then any solution of (1)-(3) in a bounded domain, with initial values near $\left(u^{*}, v^{*}\right)$, exists for 

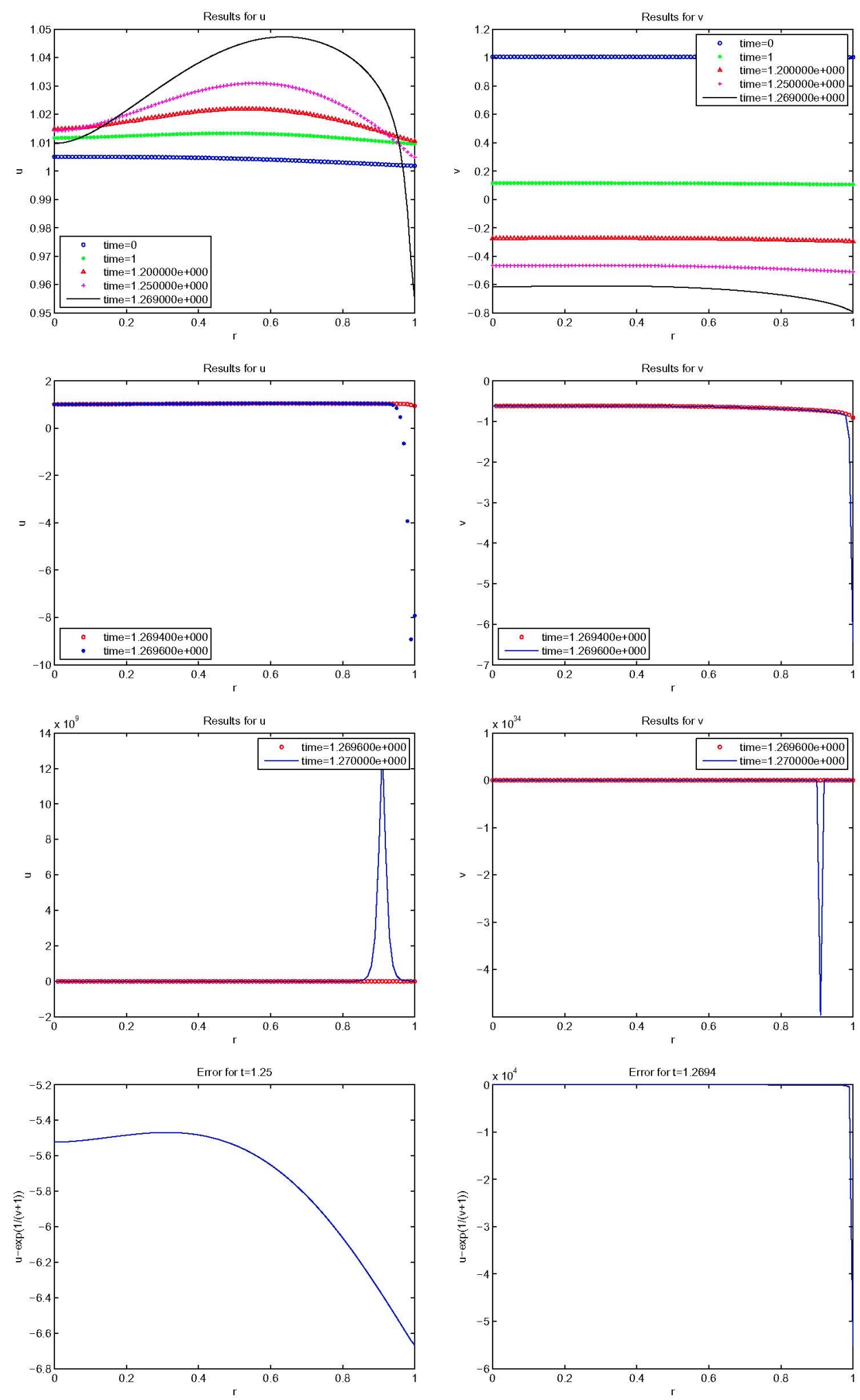

Fig. 4. Results obtained with $u_{0}(r)=v_{0}(r)=1+0.005 e^{-r^{3}}, \chi(v)=\frac{1}{1+y^{\prime}}, h(v)=e^{\frac{1}{x+1}}$ and $\mu=1$, that is $v_{t}=u-e^{\frac{1}{v+1}}$. In this case assumption (9) is not satisfied. 
Table 1

Results obtained for $u$ and $v$ with $u_{0}(r)=v_{0}(r)=1+0.005 e^{-r^{3}}$.

$\chi(\nu)=\frac{1}{1+v}, h(\nu)=v$ and $\mu=1$, for different steps in time $\Delta t$ and space $\Delta r$ for $t=0.1$ at $r=0$.

\begin{tabular}{lll}
\hline$t=0.1, r=0$ & $u$ & $v$ \\
\hline$\Delta t=1 e-04, \Delta r=1 e-2$ & 1.0049263207 & 1.0049956217 \\
$\Delta t=1 e-06, \Delta r=5 e-3$ & 1.0049274942 & 1.0049957621 \\
$\Delta t=1 e-06, \Delta r=2 e-3$ & 1.0049274269 & 1.0049957564 \\
$\Delta t=1 e-06, \Delta r=1 e-3$ & 1.0049272392 & 1.0049957383 \\
\hline
\end{tabular}

Table 2

Results obtained for $u$ and $v$ with $u_{0}(r)=v_{0}(r)=1+0.005 e^{-r^{3}}, \chi(v)=\frac{1}{1+v^{2}}, h(v)=v$ and $\mu=1$, for different steps in time $\Delta t$ and space $\Delta r$ for $t=0.1$ at $r=1$.

\begin{tabular}{lll}
\hline$t=0.1, r=1$ & $u$ & $v$ \\
\hline$\Delta t=1 e-04, \Delta r=1 e-2$ & 1.0034998369 & 1.0019458848 \\
$\Delta t=1 e-06, \Delta r=5 e-3$ & 1.0035001391 & 1.0019457786 \\
$\Delta t=1 e-06, \Delta r=2 e-3$ & 1.0035001888 & 1.0019457409 \\
$\Delta t=1 e-06, \Delta r=1 e-3$ & 1.0035002060 & 1.0019457284 \\
\hline
\end{tabular}

all $t>0$ and converges as $t \rightarrow \infty$ to a nearby stationary solution $(\bar{u}, \bar{v})$. Hence, under assumption (4), provided the initial distribution is nearly uniform, solutions tend to an uniform distribution.

A natural question is, if assumption (4) is not satisfied, how do the solutions behave? do we expect global existence or finite time blow up? is assumption (4) a necessary condition to have stability? Such questions have been the main motivation of our work.

As expected, the numerical simulations show the stabilization of solutions to the problem with radial symmetry when the assumption (4) is satisfied (in particular, it is satisfied (9), see Fig. 1). If assumption (4) (in particular (9)) is not satisfied (Figs. 3 and 4) then the solutions can stabilize or not depending on the expression of $h(v)$ in (11).

In Fig. 3, the solution stabilize to constant values for $u$ and $v$, so, the numerical simulations shows that assumption (4) is not a necessary assumption to obtain stability. Fig. 4 shows "numerical" blow up, which induces to conjecture that for some particular profiles of $g$ blow up occurs.

The numerical method described in this paper follows the ideas presented in [4] and the algorithm is based on the reformulation of the convection term in (10), the equation for $u$, in terms of the total derivative and the application of the characteristics method combined with a finite element one. This method is presented as an alternative to spectral methods, upwind algorithms and finite volume methods, which prove to be very effective to tackle models of chemotaxis-haptotaxis type (see for example [3,7] and references therein).

We would like to remark that the numerical method described here can be easily generalized and applied to other definitions of the haptotactic sensitivity $\chi$ and other expressions of $g(u, v)$, not included here for the sake of brevity. The method proves to be efficient and it reproduces the behavior expected in cases for which analytical results are known. So, the numerical method employed in this paper could be effectively used to obtain some insight about the behavior of solutions to the problem considered in [9], Example 1, but also about the behavior of solutions to systems of similar kind.

This paper can be considered as the starting point of future research involved with the application of generalizations of the scheme developed here to the study of fully 2D problems (no radial symmetry assumed) and problems which also include chemotaxis terms. Notice that, if one wants to deal with biomedical applications and therefore to obtain biologically admissible solutions then a more careful choice of the definitions for $g(u, v)$ and $\chi$ should be considered. In this occasion, we were more concerned with bringing some light to the questions above mentioned and showing that according to the results obtained with our numerical scheme, (4) is a sufficient condition but not necessary in order to obtain the stabilization of solutions, than with the modeling or biomedical applications.

\section{Acknowledgements}

The first author is partially supported by Research Projects MTM2011-26016 (MICINN) and TEC2012-39095-C03-02. The second author is partially supported by Research Project MTM2013-42907-P.

\section{Appendix A. Supplementary data}

Supplementary data associated with this article can be found, in the online version, at http://dx.doi.org/10.1016/j.amc. 2015.01.043. 


\section{References}

[1] A.R.A. Anderson, M.A.J. Chaplain, Continuous and discrete mathematical models of tumor-induced angiogenesis, Bull. Math. Biology 60 (1998) 857899.

[2] G. Bayada, M. Chambat, C. Vázquez, Characteristics method for the formulation and computation of free boundary cavitation problem, J. Comput. Appl. Math. 98 (1998) 191-212.

[3] A. Barrea, C. Turner, A numerical analysis of a model of growth tumor, Appl. Math. Comput. 167 (2005) $345-354$

[4] M. Bercovier, O. Pironneau, V. Sastri, Finite elements and characteristics for some parabolic-hyperbolic problems, Appl. Math. Model. 7 (1) (1983) $89-$ 96.

[5] N. Calvo, J.I. Díaz, J. Durany, E. Schiavi, C. Vázquez, On a doubly nonlinear parabolic obstacle problem modelling ice sheet dynamics, SIAM J. Appl. Math 63 (2) (2002) 683-707.

[6] N. Calvo, J. Durany, C. Vázquez, Numerical approach of temperature distribution in a free boundary model for polythermal ice sheets, Num. Math 83 (1999) 557-580.

[7] A. Chertock, A. Kurganov, A second-order positivity preserving central-upwind scheme for chemotaxis and haptotaxis models, Numer. Math. 111 (2008) 169-205.

[8] J. Douglas, T.F. Russel, Numerical methods for convection dominated diffusion problems based on combining the method of characteristics with finite element or finite difference procedures, SIAM J. Numer. Anal 19 (5) (1982) 871-885.

[9] A. Friedman, J.I. Tello, Stability of solutions of chemotaxis equations in reinforced random walks, J. Math Anal. Appl. 272 (2002) $138-163$.

[10] A. Gerish, M.A.J. Chaplain, Robust numerical methods for taxis-diffusion-reaction systems: applications to biomedical problems, Math. Comp. Model. $43(1-2)(2006) 49-75$.

[11] E.F. Keller, L.A. Segel, Initiation of slime mould aggregation viewed as an instability, J. Theor. Biol. 26 (1970) 399-415.

[12] H.A. Levine, B.D. Sleeman, M. Nilsen-Hamilton, Mathematical modeling of the onset of capillary formation initiating angiogenesis, J. Math. Biology 42 (2001) $195-238$.

[13] E. Logak, C. Wang, The singular limit of a haptotasis model with biestable growth, Commun. Pure Appl. Anal. 11 (1) (2012) $209-228$.

[14] H.G. Othmer, A. Stevens, Aggregation, blow up, and collapse: the ABC's of taxis in reinforced random walks, SIAM, J. Appl. Math. 57 (4) (1997) 10441081.

[15] C.S. Patlak, Random walk with persistence and external bias, Bulletin Math. Biophys. 15 (3) (1953) $311-338$

[16] Y. Tao, M. Winkler, A chemotaxis-haptotaxis model: the roles of nonlinear diffusion and logistic source, SIAM J. Math. Anal. 43 (2) (2011) 685-704. 